\title{
Preliminary study on the effect of brazilin on biofilms of Staphylococcus aureus
}

\author{
DAN PENG $^{1 *}$, ANLIN CHEN $^{1 *}$, BIN SHI $^{2}$, XUN MIN $^{1}$, TAO ZHANG $^{2}$, ZHELING DONG $^{1}$, \\ HUAN YANG $^{1}$, XIANLIAN CHEN ${ }^{1}$, YINGBIAO TIAN ${ }^{3}$ and ZEHUI CHEN ${ }^{1}$ \\ ${ }^{1}$ Department of Clinical Laboratory, The Affiliated Hospital of Zunyi Medical University, Zunyi, Guizhou 563000; \\ ${ }^{2}$ Department of Clinical Laboratory, School of Laboratory Medicine, Zunyi Medical University, Zunyi, Guizhou 563003; \\ ${ }^{3}$ Department of Pharmacy, The Affiliated Hospital of Zunyi Medical University, Zunyi, Guizhou 563000, P.R. China
}

Received December 13, 2017; Accepted June 1, 2018

DOI: $10.3892 /$ etm.2018.6403

\begin{abstract}
Biofilms significantly enhance antibiotic resistance by inhibiting penetration of antibiotics and are shielded from the immune system via the formation of an extracellular polymeric matrix. Innovative and novel approaches are required for the inhibition of biofilm formation and treatment of biofilm-associated infectious diseases. In the current study, a biofilm model of Staphylococcus aureus was established in vitro to explore inhibitory effects of brazilin (BN) on biofilm formation and to evaluate damaging effects of $\mathrm{BN}$ in the presence and absence of vancomycin (VCM) on the biofilm. Antibiofilm-infection mechanisms of $\mathrm{BN}$ were observed. In these experiments, the clinical strain of S. aureus C-4-4 was isolated for biofilm formation. Crystal violet staining and fluorescence microscopy revealed that $\mathrm{BN}$ inhibited biofilm formation in vitro and the best effect was observed with two times the minimum inhibitory concentration of BN following $48 \mathrm{~h}$ incubation. Additionally, the results demonstrated that $\mathrm{BN}$ in combination with $\mathrm{VCM}$ enhanced the damage to biofilms, whereas VCM alone did not. The results of the reverse transcription-quantitative polymerase chain reaction analyses demonstrated that $\mathrm{BN}$ downregulated gene expression of intercellular adhesion (ica) A and upregulated icaR and the quorum-sensing (QS) system regulator accessory gene regulator $\mathrm{A}$. In summary, $\mathrm{BN}$
\end{abstract}

Correspondence to: Dr Zehui Chen, Department of Clinical Laboratory, The Affiliated Hospital of Zunyi Medical University, 201 Dalian Road, Zunyi, Guizhou 563000, P.R. China

E-mail: czhtyb@163.com

Dr Yingbiao Tian, Department of Pharmacy, The Affiliated Hospital of Zunyi Medical University, 201 Dalian Road, Zunyi, Guizhou 563000, P.R. China

E-mail: tybczh@163.com

*Contributed equally

Key words: brazilin, vancomycin, Staphylococcus aureus, biofilm inhibited S. aureus biofilm formation and destroyed biofilms, while simultaneously increasing permeability to VCM. BN was able to reduce production of the extracellular polymeric matrix and inhibited the QS system. These results support the use of $\mathrm{BN}$ as a novel drug and treatment strategy for $S$. aureus biofilm-associated infections.

\section{Introduction}

Staphylococcus aureus is a Gram-positive bacterium that typically colonizes the surface of the skin and, as an important pathogen of nosocomial cross-infection, it is also a common pathogenic bacterium of biofilm infections $(1,2)$. More than $80 \%$ of human infections are caused by biofilms, including endocarditis, osteomyelitis and implant-associated infections (3). S. aureus-associated infections lead to an increase in hospital stays, in addition to hospital-associated mortality (4). $S$. aureus biofilms in humans cause chronic persistent infections that are difficult to cure and represent a great challenge for clinical treatment (5).

Biofilms may adhere to surfaces and are enclosed in a self-produced polymeric matrix, which enhances antibiotic resistance and is shielded from the immune system (6). Biofilm-associated infections are difficult to treat and these chronic or relapsing infections typically require increased drug doses or prolonged antibiotic treatment, which is associated with development of drug resistances (7). Biofilm resistance is commonly 10-1,000 times stronger than that of planktonic bacteria (8). At present, vancomycin (VCM) is the most commonly administered drug for $S$. aureus biofilm-associated infections (9). However, there is cause for concern due to recent developments of VCM-intermediate $S$. aureus and vancomycin-resistant $S$. aureus (VRSA) strains (10). Combination of VCM with rifampin, oxacillin, linezolid and tigecycline has been considered in order to improve treatment effectiveness and to reduce resistance (11). Nevertheless, studies (12-14) have indicated that although this combination may be effective against methicillin-sensitive $S$. aureus, it may not hold promise for use in treating methicillin-resistant $S$. aureus biofilm infections. Therefore, there is a requirement to identify innovative and novel approaches for the prevention of biofilm formation. 
Several studies (15-17) have reported that the use of traditional herbal medicine in the prevention and treatment of biofilm infections is advantageous. Traditional herbal medicine may not only inhibit the formation of biofilms and destroy biofilms, but it may further enhance resistance to infection when combined with antibiotics (18). At the same time, these treatments exhibit little side effects, strong pharmacological action, wide availability and do not easily lead to bacterial drug resistance (19-20). This has become another notable focus of research, distinct from antibiotics. A recent study screened 40 types of traditional herbal medicines common in the Guizhou province of China to test for minimum inhibitory concentration (MIC) for S. aureus ATCC25923 biofilms (21). The results demonstrated that Caesalpinia sappan L. has bacteriostatic properties. These findings were further analyzed in the present study.

Natural products may be utilized as raw materials in the development of novel antibacterial substances (18). Brazilin $(\mathrm{BN})$ is isolated from the traditional herbal medicine Caesalpinia sappan $L$ and is a principal active component of it (20). Evidence has indicated that BN exhibits multiple biological properties, including immune system modulatory, antioxidant, anti-inflammatory, antiplatelet, antihepatotoxicity and antitumor activities $(19,20)$. Furthermore, a number of studies have demonstrated that $\mathrm{BN}$ is associated with antimicrobial activity $(22,23)$.

The current study aimed to determine whether, and the degree to which, BN may inhibit $S$. aureus biofilm formation and increase antibiotic susceptibility. Another aim was to demonstrate whether $\mathrm{BN}$ was able to reduce secretion of extracellular polysaccharides and interfere with the quorum-sensing (QS) system. To the best of our knowledge, this has not yet been reported and requires further research.

\section{Materials and methods}

Bacterial strains. A total of 50 clinical strains of $S$. aureus were prepared. Samples were collected from The Affiliated Hospital of Zunyi Medical University (Guizhou, China) in January 2017 (Table I) and patients provided written informed consent prior to collection of blood samples and swabs. The present study was approved by the Ethics Committee of the Affiliated Hospital of Zunyi Medical University. Isolated S. aureus C-4-4 was selected as preliminary studies revealed it had the strongest ability to form biofilms, as determined by Congo red and Crystal violet staining (data not shown) and is the strain discussed subsequently. The $S$. aureus ATCC25923 standard strain (American Type Culture Collection, Manassas, VA, USA) was used as quality control. S. aureus strains were routinely cultured on blood agar (BA) plates at $37^{\circ} \mathrm{C}$ and $5 \% \mathrm{CO}_{2}$ for $24 \mathrm{~h}$. Single colonies were selected from a culture plate and the bacterial suspension was diluted in Mueller Hinton (M-H) broth (BD Difco ${ }^{\mathrm{TM}}$; BD Biosciences, Franklin Lakes, NJ, USA) to an optical density at $600 \mathrm{~nm}$ (OD600) of 0.1 prior to use.

Determination of the effect of $B N$ on S. aureus. BN (Sigma-Aldrich; Merck KGaA, Darmstadt, Germany) purity was confirmed to $>98 \%$ using high-performance liquid chromatography. BN $(1,024 \mu \mathrm{g} / \mathrm{ml})$ was dissolved in deionized water and stored at $4^{\circ} \mathrm{C}$ until use. The $\mathrm{pH}$ of the solution was maintained at 7.2-7.4. Antimicrobial susceptibility testing of $\mathrm{BN}$ and VCM was performed using the standardized broth microdilution method in 96-well U-bottom plates (Costar 3590; Corning Incorporated, Corning, NY, USA) using M-H broth, according to the 2015 Clinical and Laboratory Standards Institute guidelines (24). VCM $(0.5 \mu \mathrm{g} / \mathrm{ml}$; Sigma-Aldrich; Merck $\mathrm{KGaA}$ ) was used as a positive control.

Crystal violet staining elaborating the effect of $B N$ on $S$. aureus biofilm formation. S. aureus strains were cultured on BA plates at $37^{\circ} \mathrm{C}$ and $5 \% \mathrm{CO}_{2}$ overnight. The following day, the bacterial suspension was diluted in $\mathrm{M}-\mathrm{H}$ broth to OD600 $=0.1$. Bacterial suspension $(100 \mu \mathrm{l})$ was applied to sterile polystyrene 96 -well plates and $100 \mu \mathrm{l} \mathrm{BN}(1 / 2,1$ or 2 MIC) were added to final concentrations of $1 / 4,1 / 2$ and 1 MIC, respectively. VCM (100 $\mu 1,2$ MIC) was used as positive control and deionized water as blank. Suspensions were cultivated at $37^{\circ} \mathrm{C}$ and $5 \% \mathrm{CO}_{2}$ for $6,12,24$ and $48 \mathrm{~h}$. Unbound cells from $S$. aureus 6-, 12-, 24- and 48-h incubation plates were removed by washing with PBS (3x). The biomass of each slice was air-dried for $20 \mathrm{~min}$ at $37^{\circ} \mathrm{C}$ and stained for $20 \mathrm{~min}$ at $25^{\circ} \mathrm{C}$ with $200 \mu \mathrm{l}$ filtered $0.25 \%$ crystal violet, washed with PBS $(3 \mathrm{x})$ to remove unbound stain, dried at $37^{\circ} \mathrm{C}$ for $20 \mathrm{~min}$ and solubilized in $200 \mu 195 \%$ ethanol for $15 \mathrm{~min}$ at $25^{\circ} \mathrm{C}$. A reading at $570 \mathrm{~nm}$ using an ELISA microplate reader (Multiskan FC; Thermo Fisher Scientific, Inc., Waltham, MA, USA) was obtained. Experiments were performed six times.

Fluorescence microscopy elaborating the effect of $B N$ on S. aureus biofilm formation. A polystyrene carrier (10x10 mm) was placed in a 24 -well plate and $100 \mu 1$ bacterial suspension were applied along with $100 \mu \mathrm{l} \mathrm{BN} \mathrm{(1/2,1} \mathrm{and} 2$ MIC). VCM (100 $\mu \mathrm{l} ; 2$ MIC) was used as positive control and deionized water as blank. Plates were incubated at $37^{\circ} \mathrm{C}$ and $5 \% \mathrm{CO}_{2}$ for 6, 12, 24 and $48 \mathrm{~h}$. Unbound cells from $S$. aureus 6-, 12-, 24- and 48-h incubation plates were removed by washing with PBS (3x). Biofilms were stained with fluorescein isothiocyanate-conjugated concanavalin A (FITC-ConA) for $20 \mathrm{~min}$ at $25^{\circ} \mathrm{C}$ and washed with PBS to remove stain. Fluorescent images were obtained using BX53 fluorescence microscopy (Olympus Corporation, Tokyo, Japan).

Crystal violet staining elaborating the combined effect of $B N$ and VCM on S. aureus biofilms. Bacterial suspension $(200 \mu \mathrm{l})$ was added to 96 -well plates and cultivated at $37^{\circ} \mathrm{C}$ and $5 \%$ $\mathrm{CO}_{2}$ for $48 \mathrm{~h}$ to mature the biofilm. Plates were gently washed with PBS $(3 \mathrm{x})$. BN $(100 \mu \mathrm{l})$ with or without VCM was added to each well and incubated at $37^{\circ} \mathrm{C}$ in $5 \% \mathrm{CO}_{2}$ for $6,12,24$ or $48 \mathrm{~h}$. The final concentrations of $\mathrm{BN}$ were $1 / 2,1$ and 2 MIC (16, 32 and $64 \mu \mathrm{g} / \mathrm{ml})$ and VCM were $1 / 2,1$ and 2 MIC $(0.25,0.5$ and $1 \mu \mathrm{g} / \mathrm{ml})$. Plates were cultivated at $37^{\circ} \mathrm{C}$ for $6,12,24$ and $48 \mathrm{~h}$. Plates were washed with PBS to remove planktonic cells. The biomass of each slice was air-dried at $37^{\circ} \mathrm{C}$ for $20 \mathrm{~min}$ and stained for $20 \mathrm{~min}$ at $25^{\circ} \mathrm{C}$ with $200 \mu \mathrm{l}$ filtered $0.25 \%$ crystal violet, washed with PBS $(3 \mathrm{x})$ to remove unbound stain, dried for $20 \mathrm{~min}$ at $37^{\circ} \mathrm{C}$ and solubilized in $200 \mu 195 \%$ ethanol for $15 \mathrm{~min}$ at $25^{\circ} \mathrm{C}$. A reading at $570 \mathrm{~nm}$ using an ELISA microplate reader was obtained. Experiments were performed six times. 
Table I. Samples collected from The Affiliated Hospital of Zunyi Medical University, Zunyi, China.

\begin{tabular}{|c|c|c|c|c|c|}
\hline No & Strain_ID & Age & Sex & Infection site & Month and year \\
\hline 1 & C-3-077 & 2 & Male & Wound & Jan-17 \\
\hline 2 & C-3-078 & 1 & Female & Wound & Jan-17 \\
\hline 3 & C-3-079 & 54 & Male & Abscess & Jan-17 \\
\hline 4 & C-4-013 & 23 & Female & Respiratory & Jan-17 \\
\hline 5 & C-3-080 & 8 & Male & Respiratory & Jan-17 \\
\hline 6 & C-3-081 & 23 & Female & Abscess & Jan-17 \\
\hline 7 & C-3-082 & 55 & Female & Respiratory & Jan-17 \\
\hline 8 & C-3-083 & 41 & Male & Wound & Jan-17 \\
\hline 9 & C-3-084 & 33 & Male & Wound & Jan-17 \\
\hline 10 & C-3-085 & 37 & Male & Respiratory & Jan-17 \\
\hline 11 & C-3-086 & 3 & Female & Wound & Jan-17 \\
\hline 12 & C-3-087 & 39 & Female & Abscess & Jan-17 \\
\hline 13 & C-3-088 & 21 & Female & Abscess & Jan-17 \\
\hline 14 & C-3-089 & 2 & Male & Wound & Jan-17 \\
\hline 15 & C-3-090 & 0 & Male & Respiratory & Jan-17 \\
\hline 16 & C-3-091 & 64 & Female & Respiratory & Jan-17 \\
\hline 17 & C-3-092 & 50 & Male & Wound & Jan-17 \\
\hline 18 & C-3-093 & 57 & Female & Respiratory & Jan-17 \\
\hline 19 & C-3-094 & 75 & Male & Respiratory & Jan-17 \\
\hline 20 & C-3-095 & 0 & Female & Abscess & Jan-17 \\
\hline 21 & C-3-096 & 52 & Male & Respiratory & Jan-17 \\
\hline 22 & C-3-097 & 63 & Male & Respiratory & Jan-17 \\
\hline 23 & C-3-098 & 3 & Female & Wound & Jan-17 \\
\hline 24 & C-3-099 & 23 & Female & Abscess & Jan-17 \\
\hline 25 & C-3-100 & 2 & Female & Wound & Jan-17 \\
\hline 26 & C-4-001 & 0 & Male & Respiratory & Jan-17 \\
\hline 27 & C-4-020 & 71 & Female & Respiratory & Jan-17 \\
\hline 28 & C-4-003 & 0 & Female & Respiratory & Jan-17 \\
\hline 29 & C-4-004 & 46 & Female & Abscess & Jan-17 \\
\hline 30 & C-4-005 & 4 & Female & Wound & Jan-17 \\
\hline 31 & C-4-006 & 47 & Male & Wound & Jan-17 \\
\hline 32 & C-4-007 & 50 & Male & Wound & Jan-17 \\
\hline 33 & C-4-008 & 39 & Male & Wound & Jan-17 \\
\hline 34 & C-4-009 & 60 & Female & Wound & Jan-17 \\
\hline 35 & C-4-010 & 0 & Female & Respiratory & Jan-17 \\
\hline 36 & C-4-011 & 1 & Male & Wound & Jan-17 \\
\hline 37 & C-4-012 & 64 & Female & Wound & Jan-17 \\
\hline 38 & D-1-078 & 45 & Male & Wound & Jan-17 \\
\hline 39 & D-1-079 & 19 & Male & Abscess & Jan-17 \\
\hline 40 & D-1-080 & 5 & Female & Bacteremia & Jan-17 \\
\hline 41 & D-1-081 & 48 & Male & Respiratory & Jan-17 \\
\hline 42 & D-1-082 & 37 & Male & Abscess & Jan-17 \\
\hline 43 & D-1-083 & 51 & Male & Wound & Jan-17 \\
\hline 44 & D-1-084 & 51 & Male & Abscess & Jan-17 \\
\hline 45 & D-1-085 & 5 & Male & Bacteremia & Jan-17 \\
\hline 46 & D-1-086 & 51 & Male & Wound & Jan-17 \\
\hline 47 & D-1-087 & 75 & Male & Respiratory & Jan-17 \\
\hline 48 & D-1-088 & 41 & Male & Cerebrospinal fluid & Jan-17 \\
\hline 49 & D-1-089 & 49 & Male & Bacteremia & Jan-17 \\
\hline 50 & D-1-090 & 59 & Female & Cerebrospinal fluid & Jan-17 \\
\hline
\end{tabular}


Table II. Effect of BN on S. aureus biofilm formation measured using crystal violet staining.

\begin{tabular}{lllll}
\hline & \multicolumn{3}{c}{ OD $_{570}$} \\
\cline { 2 - 5 } Group & \multicolumn{1}{c}{$6 \mathrm{~h}$} & $12 \mathrm{~h}$ & $24 \mathrm{~h}$ & $48 \mathrm{~h}$ \\
\hline Control & $0.274 \pm 0.056$ & $0.439 \pm 0.059$ & $0.726 \pm 0.119$ & $1.133 \pm 0.283$ \\
1 MIC VCM & $0.195 \pm 0.030^{\mathrm{a}}$ & $0.274 \pm 0.053^{\mathrm{a}}$ & $0.356 \pm 0.066^{\mathrm{a}}$ & $0.470 \pm 0.125^{\mathrm{a}}$ \\
$1 / 4 \mathrm{MIC} \mathrm{BN}$ & $0.244 \pm 0.049$ & $0.336 \pm 0.012$ & $0.535 \pm 0.099^{\mathrm{a}}$ & $0.672 \pm 0.163^{\mathrm{a}}$ \\
$1 / 2 \mathrm{MIC} \mathrm{BN}$ & $0.221 \pm 0.028^{\mathrm{a}}$ & $0.308 \pm 0.064^{\mathrm{a}}$ & $0.441 \pm 0.095^{\mathrm{a}}$ & $0.510 \pm 0.108^{\mathrm{a}}$ \\
1 MIC BN & $0.202 \pm 0.033^{\mathrm{a}}$ & $0.281 \pm 0.075^{\mathrm{a}}$ & $0.346 \pm 0.127^{\mathrm{a}}$ & $0.450 \pm 0.121^{\mathrm{a}}$ \\
\hline
\end{tabular}

${ }^{\mathrm{a}} \mathrm{P}<0.05$ vs. control. BN, brazilin; VCM, vancomycin; MIC, minimum inhibitory concentration; OD, optical density.

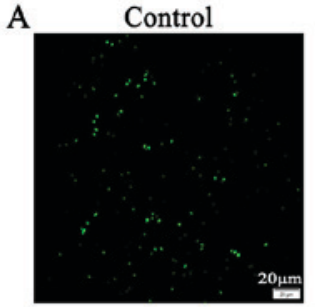

B

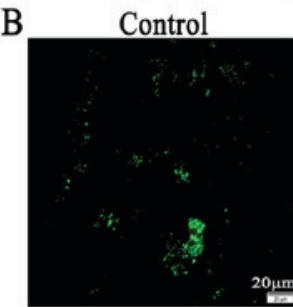

C
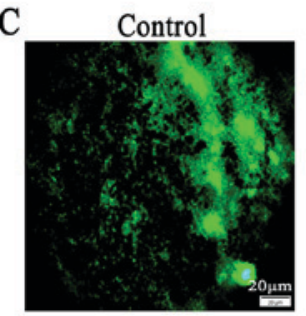

D

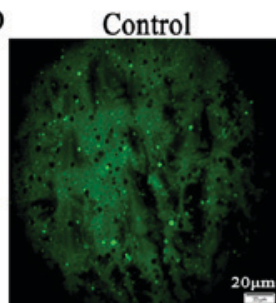

1 MIC VCM

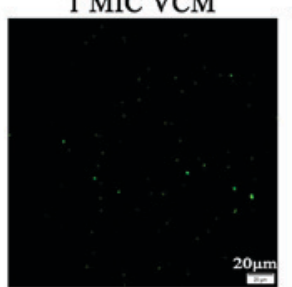

1 MIC VCM

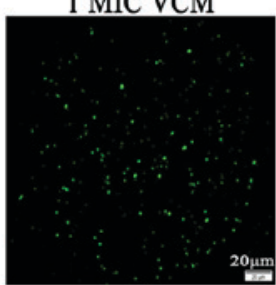

1 MIC VCM

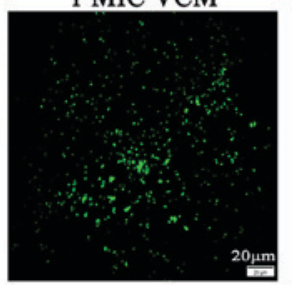

1 MIC VCM

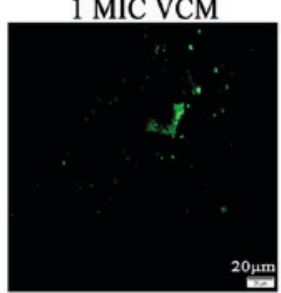

1/4 MIC BN

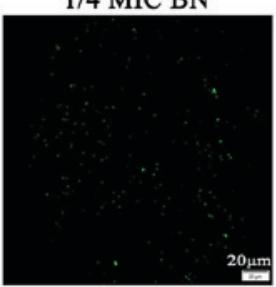

1/4 MIC BN

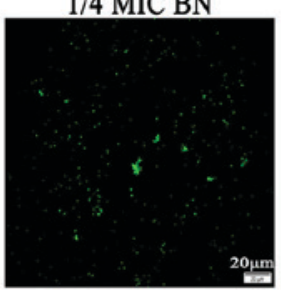

1/4 MIC BN

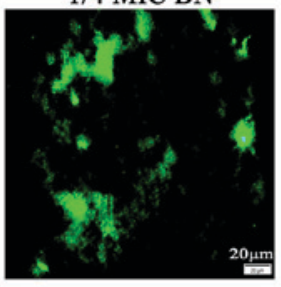

1/4 MIC BN

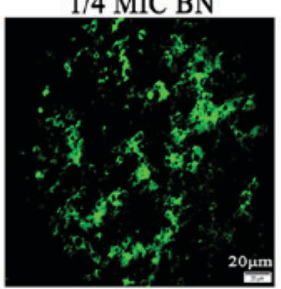

1/2 MIC BN

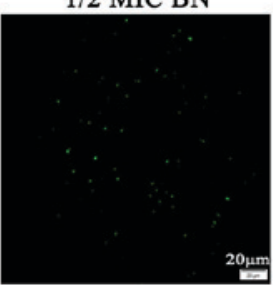

1/2 MIC BN

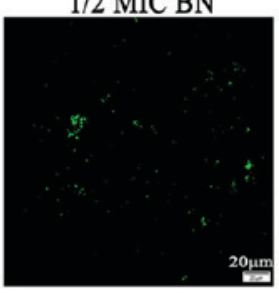

$1 / 2$ MIC BN

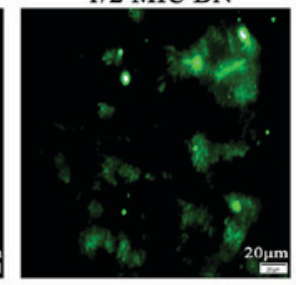

1/2 MIC BN

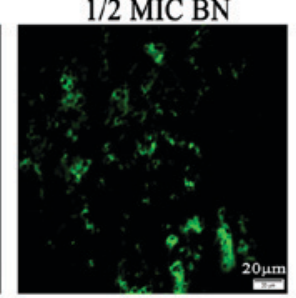

$1 \mathrm{MIC} \mathrm{BN}$

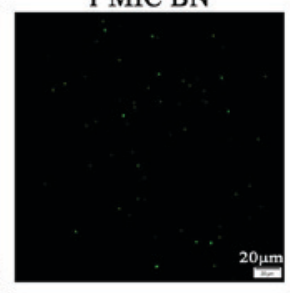

1 MIC BN

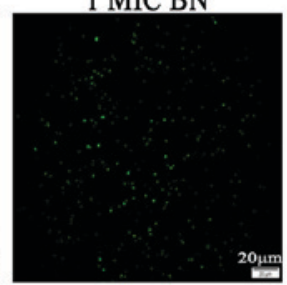

1 MIC BN

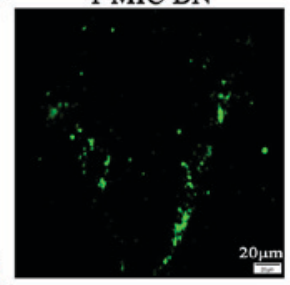

1 MIC BN

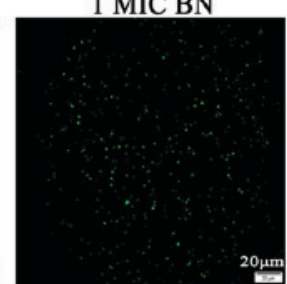

Figure 1. Fluorescence microscopy based on interactions with polysaccharides of the $S$. aureus biofilm (magnification, x400). Experiments used fluorescein isothiocyanate-conjugated concanavalin A to evaluate the effect of BN at 1/4,1/2 and 1 MIC on biofilm formation following treatment for (A) 6, (B) 12, (C) 24 and (D) $48 \mathrm{~h}$. Scale bars, $20 \mu \mathrm{m}$. BN, brazilin; MIC, minimum inhibitory concentration; VCM, vancomycin.

Fluorescence microscopy elaborating the combined effect of $B N$ and VCM on S. aureus biofilms. Biofilms were prepared in 24-well plates containing a polystyrene slice as described. Slices were gently washed with PBS (2x). BN (100 $\mu 1)$ with or without VCM was added to each well. Concentrations of BN were 1/2, 1 and $2 \mathrm{MIC}(16,32$ and $64 \mu \mathrm{g} / \mathrm{ml})$ and $\mathrm{VCM}$ were $1 / 2,1$ and $2 \operatorname{MIC}(0.25,0.5$ and $1 \mu \mathrm{g} / \mathrm{ml})$. Plates were incubated at $37^{\circ} \mathrm{C}$ for $6,12,24$ and $48 \mathrm{~h}$. Slices were washed with PBS to remove planktonic cells. Biofilms were stained with FITC-ConA for $20 \mathrm{~min}$ at $25^{\circ} \mathrm{C}$ and washed with PBS to remove stain. Fluorescent images were captured by fluorescence microscopy. Experiments were performed in triplicate.

Biofilm bacterial colony-forming unit (CFU) counts. Bacterial suspension $(200 \mu \mathrm{l})$ was added to 96 -well plates and cultivated at $37^{\circ} \mathrm{C}$ and $5 \% \mathrm{CO}_{2}$ for $48 \mathrm{~h}$ to mature the biofilms. The 
Table III. Effect of BN combined with VCM on S aureus biofilms analyzed using crystal violet staining.

\begin{tabular}{|c|c|c|c|c|}
\hline \multirow[b]{2}{*}{ Group } & \multicolumn{4}{|c|}{$\mathrm{OD}_{570}$} \\
\hline & $6 \mathrm{~h}$ & $12 \mathrm{~h}$ & $24 \mathrm{~h}$ & $48 \mathrm{~h}$ \\
\hline Blank & $1.224 \pm 0.246$ & $1.291 \pm 0253$ & $1.226 \pm 0.194$ & $1.283 \pm 0.272$ \\
\hline 1/2 MIC VCM & $1.197 \pm 0.262$ & $1.269 \pm 0.182$ & $1.168 \pm 0.234$ & $1.156 \pm 0.154$ \\
\hline $1 \mathrm{MIC}$ VCM & $1.211 \pm 0.287$ & $1.264 \pm 0.267$ & $1.151 \pm 0.163$ & $1.112 \pm 0.116$ \\
\hline $2 \mathrm{MIC}$ VCM & $1.140 \pm 0.296$ & $1.224 \pm 0.311$ & $1.105 \pm 0.121$ & $1.041 \pm 0.136^{\mathrm{a}}$ \\
\hline $1 / 2 \mathrm{MIC} \mathrm{BN}$ & $1.211 \pm 0.291$ & $1.021 \pm 0.180$ & $0.961 \pm 0.067^{\mathrm{a}}$ & $0.882 \pm 0.121^{\mathrm{a}}$ \\
\hline $1 \mathrm{MIC} \mathrm{BN}$ & $1.112 \pm 0.287$ & $1.048 \pm 0.196$ & $0.904 \pm 0.127^{\mathrm{a}, \mathrm{c}}$ & $0.810 \pm 0.134^{\mathrm{a}, \mathrm{c}}$ \\
\hline $2 \mathrm{MIC} \mathrm{BN}$ & $0.965 \pm 0.134^{\mathrm{a}}$ & $0.965 \pm 0.188^{\mathrm{a}}$ & $0.877 \pm 0.176^{\mathrm{a}, \mathrm{c}}$ & $0.711 \pm 0.201^{\mathrm{a}, \mathrm{c}}$ \\
\hline 1/2 MIC VCM+1/2 MIC BN & $0.796 \pm 0.060^{\mathrm{a}}$ & $0.916 \pm 0.160^{\mathrm{a}}$ & $0.854 \pm 0.121^{\mathrm{a}, \mathrm{c}}$ & $0.747 \pm 0.146^{\mathrm{a}, \mathrm{c}}$ \\
\hline 1/2 MIC VCM+1 MIC BN & $0.945 \pm 0.149^{\mathrm{a}, \mathrm{c}}$ & $0.880 \pm 0.075^{\mathrm{a}, \mathrm{c}}$ & $0.802 \pm 0.876^{\mathrm{a}, \mathrm{c}}$ & $0.678 \pm 0.173^{\mathrm{a}, \mathrm{c}}$ \\
\hline 1/2 MIC VCM+2 MIC BN & $0.903 \pm 0.159^{\mathrm{a}, \mathrm{c}}$ & $0.857 \pm 0.160^{\mathrm{a}, \mathrm{c}}$ & $0.772 \pm 0.169^{\mathrm{a}, \mathrm{c}}$ & $0.644 \pm 0.170^{\mathrm{a}, \mathrm{c}}$ \\
\hline $1 \mathrm{MIC} \mathrm{VCM}+1 / 2 \mathrm{MIC} \mathrm{BN}$ & $0.882 \pm 0.148^{\mathrm{a}, \mathrm{c}}$ & $0.833 \pm 0.211^{\mathrm{a}, \mathrm{c}}$ & $0.757 \pm 0.120^{\mathrm{a}, \mathrm{c}}$ & $0.584 \pm 0.214^{\mathrm{a}-\mathrm{c}}$ \\
\hline $1 \mathrm{MIC} \mathrm{VCM}+1 \mathrm{MIC} \mathrm{BN}$ & $0.863 \pm 0.219^{\mathrm{a}-\mathrm{c}}$ & $0.805 \pm 0.177^{\mathrm{a}-\mathrm{c}}$ & $0.720 \pm 0.170^{\mathrm{a}-\mathrm{c}}$ & $0.526 \pm 0.222^{\mathrm{a}-\mathrm{c}}$ \\
\hline $1 \mathrm{MIC} \mathrm{VCM}+2 \mathrm{MIC} \mathrm{BN}$ & $0.833 \pm 0.199^{\mathrm{a}-\mathrm{c}}$ & $0.816 \pm 0.070^{\mathrm{a}-\mathrm{c}}$ & $0.654 \pm 0.182^{\mathrm{a}-\mathrm{c}}$ & $0.498 \pm 0.144^{\mathrm{a}-\mathrm{c}}$ \\
\hline $2 \mathrm{MIC}$ VCM+1/2 MIC BN & $0.831 \pm 0.150^{\mathrm{a}-\mathrm{c}}$ & $0.776 \pm 0.093^{\mathrm{a}-\mathrm{c}}$ & $0.597 \pm 0.138^{\mathrm{a}-\mathrm{c}}$ & $0.469 \pm 0.158^{\mathrm{a}-\mathrm{c}}$ \\
\hline $2 \mathrm{MIC} \mathrm{VCM}+1 \mathrm{MIC}$ BN & $0.768 \pm 0.149^{\mathrm{a}-\mathrm{c}}$ & $0.732 \pm 0.157^{\mathrm{a}-\mathrm{c}}$ & $0.553 \pm 0.223^{\mathrm{a}-\mathrm{c}}$ & $0.404 \pm 0.171^{\mathrm{a}-\mathrm{c}}$ \\
\hline $2 \mathrm{MIC} \mathrm{VCM}+2 \mathrm{MIC} \mathrm{BN}$ & $0.756 \pm 0.112^{\mathrm{a}-\mathrm{c}}$ & $0.645 \pm 0.116^{\mathrm{a}-\mathrm{c}}$ & $0.507 \pm 0.209^{a-c}$ & $0.390 \pm 0.160^{\mathrm{a}-\mathrm{c}}$ \\
\hline
\end{tabular}

${ }^{\mathrm{a}} \mathrm{P}<0.05$ vs. control; ${ }^{\mathrm{b}} \mathrm{P}<0.05$ vs. $1 / 2$ and $1 \mathrm{MIC} \mathrm{BN}$; ${ }^{\mathrm{P}}<0.05$ vs. VCM. BN, brazilin; VCM, vancomycin; MIC, minimum inhibitory concentration; OD, optical density.

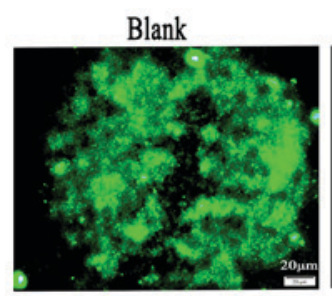

$1 / 2$ MIC BN
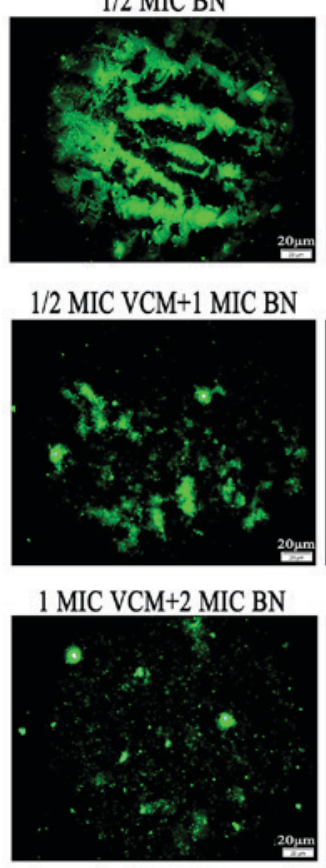

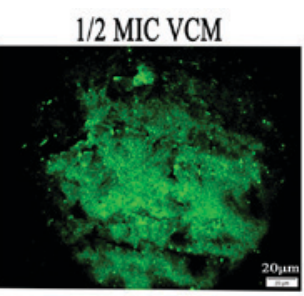

$1 \mathrm{MIC}$ BN

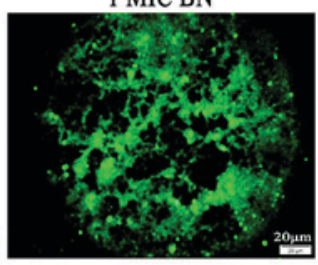

$1 / 2$ MIC VCM+2 MIC BN

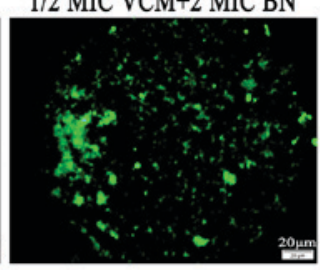

2 MIC VCM+1/2 MIC BN

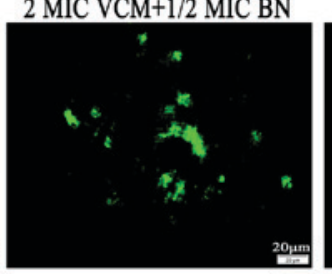

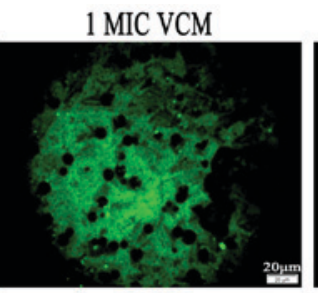

2 MIC BN

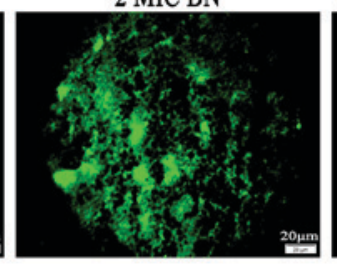

$1 \mathrm{MIC}$ VCM+1/2 MIC BN

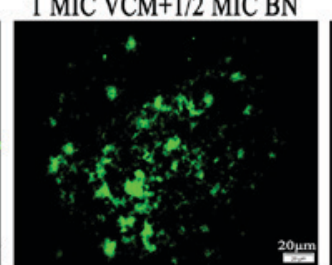

2 MIC VCM+1 MIC BN

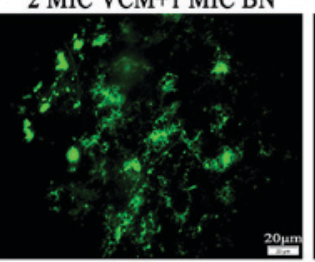

2 MIC VCM

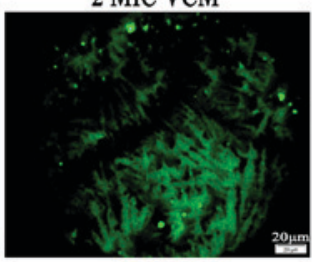

$1 / 2 \mathrm{MIC}$ VCM+1/2 MIC BN

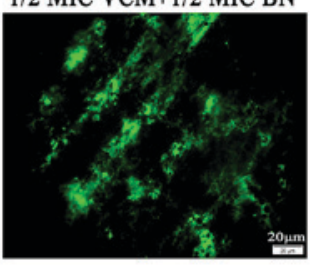

$1 \mathrm{MIC}$ VCM+1 MIC BN

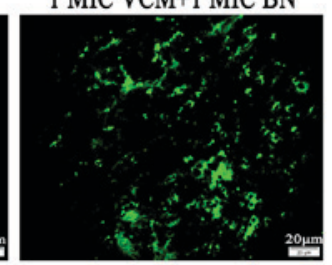

2 MIC VCM+ 2 MIC BN

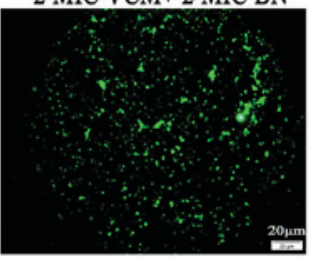

Figure 2. Fluorescence microscopy highlights effects of BN and VCM on S. aureus biofilms (magnification, x400). Effects of varying concentrations of BN $(1 / 2,1$ and $2 \mathrm{MIC})$ in the presence and absence of VCM $(1 / 2,1$ and $2 \mathrm{MIC})$ on mature biofilms were evaluated for $48 \mathrm{~h}$. Biofilms were stained with fluorescein isothiocyanate-conjugated concanavalin A. Scale bars, $20 \mu \mathrm{m}$. BN, brazilin; VCM, vancomycin; MIC, minimum inhibitory concentration. 
Table IV. Influence of BN and VCM of bacteria counts.

$\mathrm{x} 10^{6}$ colony-forming unit $/ \mathrm{ml}$

\begin{tabular}{lcccc}
\cline { 2 - 5 } Group & $6 \mathrm{~h}$ & $12 \mathrm{~h}$ & $24 \mathrm{~h}$ & $48 \mathrm{~h}$ \\
\hline Blank & $256.667 \pm 24.502$ & $255.000 \pm 27.074$ & $256.333 \pm 14.503$ & $256.333 \pm 13.204$ \\
$1 / 2$ MIC VCM & $239.667 \pm 27.502$ & $246.333 \pm 30.534$ & $248.667 \pm 18.148$ & $241.333 \pm 21.221$ \\
1 MIC VCM & $241.667 \pm 10.017$ & $242.333 \pm 30.616$ & $239.000 \pm 13.454$ & $233.333 \pm 31.390$ \\
2 MIC VCM & $239.667 \pm 23.029$ & $238.000 \pm 19.519$ & $236.333 \pm 8.622$ & $199.000 \pm 19.313^{\mathrm{a}}$ \\
$1 / 2$ MIC BN & $229.333 \pm 16.563$ & $226.667 \pm 23.007$ & $215.333 \pm 12.897^{\mathrm{a}}$ & $198.333 \pm 5.695^{\mathrm{a}}$ \\
1 MIC BN & $223.000 \pm 11.136$ & $216.667 \pm 8.737^{\mathrm{a}}$ & $206.333 \pm 7.506^{\mathrm{a} c \mathrm{c}}$ & $168.667 \pm 12.583^{\mathrm{a}, \mathrm{c}}$ \\
2 MIC BN & $196.333 \pm 16.503^{\mathrm{a}, \mathrm{c}}$ & $194.000 \pm 31.241^{\mathrm{a}, \mathrm{c}}$ & $180.333 \pm 14.048^{\mathrm{a}-\mathrm{c}}$ & $116.000 \pm 14.799^{\mathrm{a}-\mathrm{c}}$ \\
$1 / 2$ MIC VCM+1/2 MIC BN & $200.667 \pm 2.517^{\mathrm{a}, \mathrm{c}}$ & $192.000 \pm 17.692^{\mathrm{a}, \mathrm{c}}$ & $182.000 \pm 19.313^{\mathrm{acc}}$ & $113.000 \pm 17.692^{\mathrm{a}-\mathrm{c}}$ \\
$1 / 2$ MIC VCM+1 MIC BN & $184.667 \pm 26.764^{\mathrm{a}-\mathrm{c}}$ & $170.000 \pm 33.151^{\mathrm{a}-\mathrm{c}}$ & $154.000 \pm 13.454^{\mathrm{a}-\mathrm{c}}$ & $109.667 \pm 9.609^{\mathrm{a}-\mathrm{c}}$ \\
$1 / 2$ MIC VCM+2 MIC BN & $176.667 \pm 15.011^{\mathrm{a}-\mathrm{c}}$ & $163.000 \pm 26.665^{\mathrm{a}-\mathrm{c}}$ & $144.667 \pm 13.577^{\mathrm{a}-\mathrm{c}}$ & $100.667 \pm 13.317^{\mathrm{a}-\mathrm{c}}$ \\
1 MIC VCM+1/2 MIC BN & $177.000 \pm 10.583^{\mathrm{a}-\mathrm{c}}$ & $156.000 \pm 12.530^{\mathrm{a}-\mathrm{c}}$ & $137.000 \pm 13.454^{\mathrm{a}-\mathrm{c}}$ & $95.667 \pm 9.074^{\mathrm{a}-\mathrm{c}}$ \\
1 MIC VCM+1 MIC BN & $168.667 \pm 20.550^{\mathrm{a}-\mathrm{c}}$ & $149.667 \pm 17.474^{\mathrm{a}-\mathrm{c}}$ & $128.667 \pm 16.258^{\mathrm{a}-\mathrm{c}}$ & $84.333 \pm 10.408^{\mathrm{a}-\mathrm{c}}$ \\
1 MIC VCM+2 MIC BN & $146.333 \pm 23.029^{\mathrm{a}-\mathrm{c}}$ & $143.000 \pm 23.516^{\mathrm{a}-\mathrm{c}}$ & $111.333 \pm 15.275^{\mathrm{acc}}$ & $70.333 \pm 15.308^{\mathrm{a}-\mathrm{c}}$ \\
2 MIC VCM+1/2 MIC BN & $141.667 \pm 35.726^{\mathrm{a}-\mathrm{c}}$ & $135.667 \pm 11.930^{\mathrm{a}-\mathrm{c}}$ & $104.333 \pm 13.868^{\mathrm{a}-\mathrm{c}}$ & $61.000 \pm 10.536^{\mathrm{a}-\mathrm{c}}$ \\
2 MIC VCM+1 MIC BN & $140.333 \pm 23.116^{\mathrm{a}-\mathrm{c}}$ & $127.000 \pm 5.292^{\mathrm{a}-\mathrm{c}}$ & $96.000 \pm 7.937^{\mathrm{acc}}$ & $56.33 \pm 9.866^{\mathrm{a}-\mathrm{c}}$ \\
2 MIC VCM+2 MIC BN & $135.667 \pm 29.143^{\mathrm{a}-\mathrm{c}}$ & $119.000 \pm 17.578^{\mathrm{a}-\mathrm{c}}$ & $83.000 \pm 16.371^{\mathrm{a}-\mathrm{c}}$ & $47.333 \pm 11.604^{\mathrm{a}-\mathrm{c}}$ \\
\hline
\end{tabular}

${ }^{\mathrm{a}} \mathrm{P}<0.05$ vs. control; ${ }^{\mathrm{b}} \mathrm{P}<0.05$ vs. $1 / 2$ and $1 \mathrm{MIC} \mathrm{BN}$; ${ }^{\mathrm{P}}<0.05$ vs. VCM. BN, brazilin; VCM, vancomycin; MIC, minimum inhibitory concentration.

mature biofilms were treated with $100 \mu 11 / 2,1$ and 2 MIC BN with or without $1 / 2,1$ and $2 \mathrm{MIC}$ VCM and incubated at $37^{\circ} \mathrm{C}$ for 6, 12, 24 and $48 \mathrm{~h}$. Slices were washed with PBS to remove planktonic cells and sonicated to disrupt cell clumps. Biofilm bacterial CFU counts were conducted by plating serial dilutions on TSB agar. Experiments were performed in triplicate.

Confocal laser scanning microscopy (CLSM). The biofilm was determined using a double live/dead staining kit (L13152 LIVE/DEAD ${ }^{\circledR}$ BacLight $^{\mathrm{TM}}$ Bacterial Viability kits; Thermo Fisher Scientific, Inc.), containing nucleic acid stains SYTO9 (green) and propidium iodide (PI, red), which differ in their ability to penetrate healthy bacterial cells. SYTO9 stain labels live bacteria, whereas PI penetrates bacteria with damaged membranes. Bacterial suspension $(4 \mathrm{ml})$ was added to the bottom of a 24 -well plate. Following $48 \mathrm{~h}$ incubation at $37^{\circ} \mathrm{C}$, plates were washed with PBS and 1/2, 1 and $2 \mathrm{MIC} B N$ with or without $1 / 2,1$ and 2 MIC VCM was added followed by incubation at $37^{\circ} \mathrm{C}$ for $48 \mathrm{~h}$. Slices were washed with PBS. According to the manufacturer's protocol, the slices were stained with SYTO9/PI for $20 \mathrm{~min}$ at $25^{\circ} \mathrm{C}$ in the dark. A TCS SP5 microscope (Leica Microsystems GmbH, Wetzlar, Germany) was used to investigate $S$. aureus biofilms.

Scanning electron microscopy (SEM). Polystyrene plates $(24 \times 24 \mathrm{~mm})$ were sterilized and placed into 6-well plates for $48 \mathrm{~h}$ to form the biofilm. BN (2 MIC, $64 \mu \mathrm{g} / \mathrm{ml}$ ) with or without VCM (2 MIC, $1 \mu \mathrm{g} / \mathrm{ml})$ were added to each well, followed by incubation at $37^{\circ} \mathrm{C}$ for $48 \mathrm{~h}$. Slices were washed with PBS and fixed at $4^{\circ} \mathrm{C}$ with $2.5 \%$ glutaraldehyde for $8 \mathrm{~h}$. Surfaces were rinsed three times and fixed with $0.1 \%$ osmium tetraoxide for $1 \mathrm{~h}$. Samples were dehydrated through a graded ethanol series
(30, 50, 70, 80, 90, 95 and $100 \%$ ) for 15 min each at room temperature and coated with gold. Images were obtained using an SU8010 SEM (Hitachi, Ltd., Tokyo, Japan) (25).

Reverse transcription-quantitative polymerase chain reaction (RT-qPCR) analysis of genes forming biofilms. Biofilms were prepared and exposed to $\mathrm{BN}$ with or without VCM in 6-well plates as described previously. Total RNA was extracted using the MiniBEST Universal RNA Extraction kit (Takara Biotechnology Co., Ltd., Dalian, China) according to the manufacturer's protocol. Biofilms were centrifuged at $7,104 \mathrm{x} \mathrm{g}$ at $4^{\circ} \mathrm{C}$ for $2 \mathrm{~min}, 600 \mu \mathrm{l}$ Buffer RL ( Takara Biotechnology Co., Ltd.) were added and incubated for 2 min at room temperature. The pyrolysis liquid was transferred into a gDNA Eraser Spin Column and centrifuged at $13,400 \mathrm{x} g$ at $4^{\circ} \mathrm{C}$ for $1 \mathrm{~min}$. To the filtrate an equal amount of $70 \%$ ethanol was added. Immediately, the mixture was transferred into an RNA Spin Column and centrifuged at $13,400 \times \mathrm{g}$ at $4^{\circ} \mathrm{C}$ for 1 min. Buffer RWA $(500 \mu \mathrm{l})$ and Buffer RWB $(600 \mu \mathrm{l})$ were added to a RNA Spin Column and spun at $13,400 \times \mathrm{g}$ at $4^{\circ} \mathrm{C}$ for $30 \mathrm{sec}$. RNA pellets were suspended in $100 \mu \mathrm{l}$ RNase-free water. cDNA was synthesized using EasyScript ${ }^{\circledR}$ One-Step gDNA Removal and cDNA Synthesis SuperMix kit (Generon, Slough, UK). RT reaction mixtures contained total RNA sample $(5 \mu \mathrm{l}$,) anchored Oligo(dT)18 Primer $(0.5 \mu \mathrm{g} / \mu \mathrm{l} ; 1 \mu \mathrm{l})$, 2X ES Reaction mix $(10 \mu \mathrm{l})$, EasyScript ${ }^{\circledR}$ RT/RI Enzyme mix (1 $\mu \mathrm{l})$, gDNA Remover $(1 \mu \mathrm{l})$ and RNase-free water to a final volume of $20 \mu \mathrm{l}$. Reverse transcriptase was inactivated by incubation at $42^{\circ} \mathrm{C}$ for $15 \mathrm{~min}$, then $85^{\circ} \mathrm{C}$ for $5 \mathrm{sec}$, followed by cooling at $4^{\circ} \mathrm{C}$ and storage at $-80^{\circ} \mathrm{C}$. Primer sequences were as follows: Intercellular adhesion (ica)A, (189 bp) forward 5'-GCA GTTGTCGATGTTGGCTA-3' and reverse 5'-TACTTCGTG 

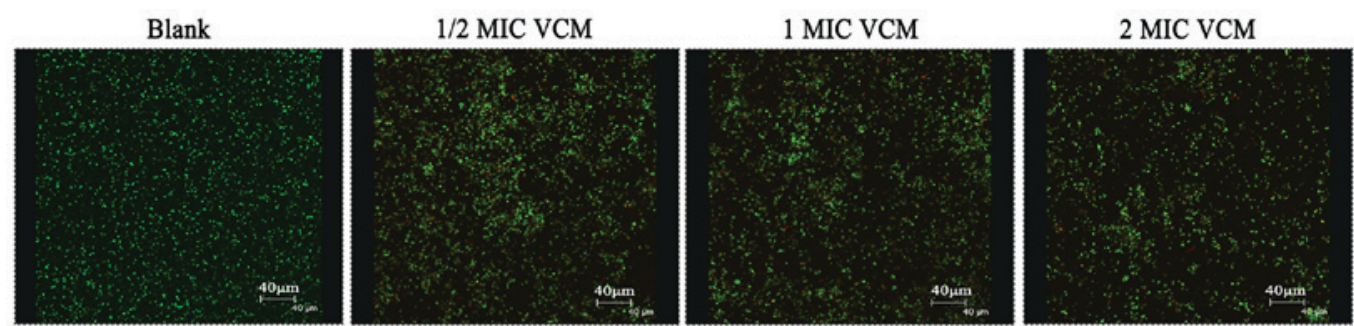

$1 / 2$ MIC BN 1 MIC BN 2 MIC BN
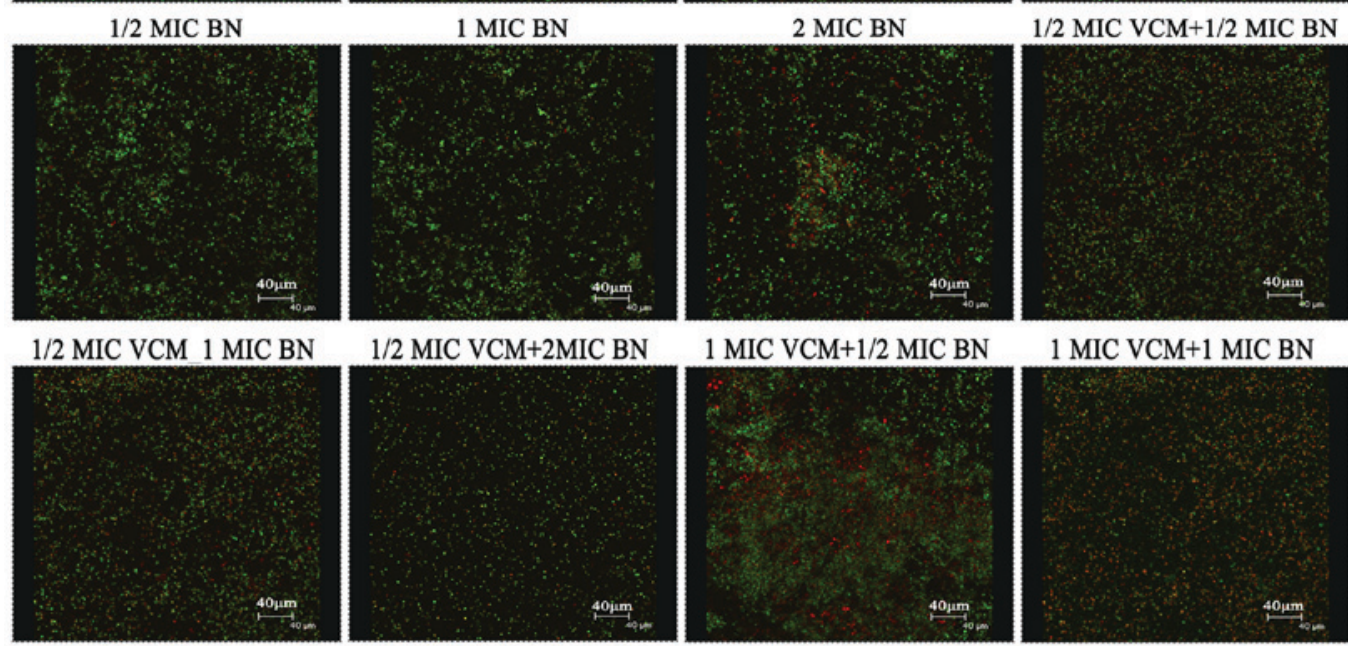

$1 / 2$ MIC VCM+2MIC BN

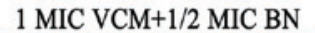

1 MIC VCM+1 MIC BN
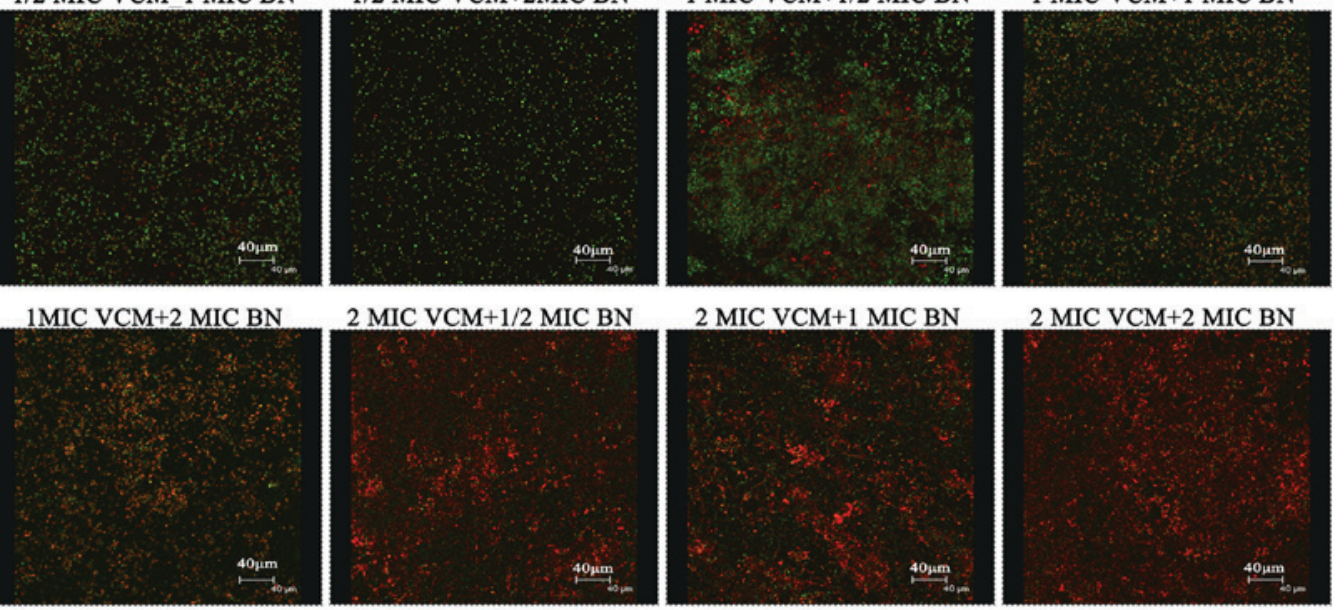

2 MIC VCM+1 MIC BN

2 MIC VCM +2 MIC BN
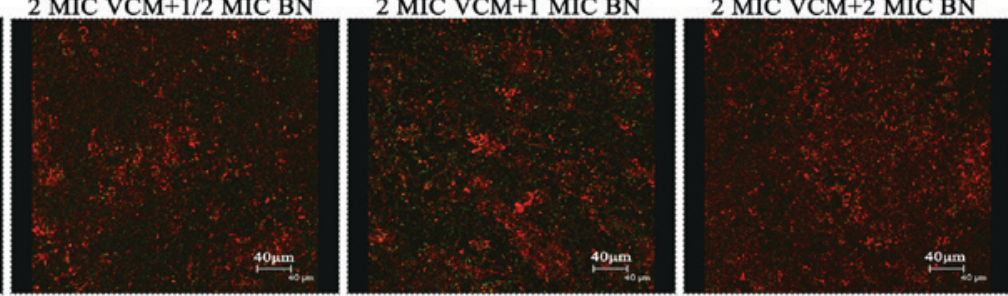

Figure 3. Biofilm assessment using confocal laser scanning microscopy (magnification, x400). Mature biofilms were stained with SYTO9/propidium iodide. Effects of varying concentrations of BN (1/2,1 and 2 MIC) in the presence and absence of VCM $(1 / 2,1$ and 2 MIC) on mature biofilms were evaluated following $48 \mathrm{~h}$ treatment. Green fluorescence represents live and red fluorescence represents dead cells. Scale bars, $40 \mu \mathrm{m}$. BN, brazilin; VCM, vancomycin; MIC, minimum inhibitory concentration.

TCCCCCTTGAG-3'; icaR (186 bp), forward 5'-TACGCCTGA GGAATTTTCTG-3' and reverse 5'-CCAAATTTTTGCGAA AAGGA-3'; accessory gene regulator (agr) A (82 bp), forward 5'-ACGTGGCAGTAATTCAGTGTATGTT-3' and reverse 5'-GGCAATGAGTCTGTGAGATTTTGT-3'; and 16SrRNA (201 bp), forward 5'-TATTTTTCCGGTTGGTCGTC-3' and reverse 5'-GGCTTAACCTTGCCATCAGA-3'. qPCR was performed according to the manufacturer's protocol (Takara Biotechnology Co., Ltd.), in $20 \mu$ reactions, containing SYBR Premix Ex TaqII $(10 \mu \mathrm{l})$, forward and reverse primers $(0.8 \mu \mathrm{l})$, cDNA $(2 \mu \mathrm{l})$, ROX Reference Dye II $(0.4 \mu \mathrm{l})$ and deionized water $(6 \mu 1)$. An Applied Biosystems 7500 Fast Real-Time PCR system (Applied Biosystems, Thermo Fisher Scientific, Inc.) was used. Cycling parameters were as follows: $95^{\circ} \mathrm{C}$ for $30 \mathrm{sec} ; 95^{\circ} \mathrm{C}$ for $5 \mathrm{sec}$ and $60^{\circ} \mathrm{C}$ for $34 \mathrm{sec}$ for 40 cycles and one melt curve at $95^{\circ} \mathrm{C}$ for $15 \mathrm{sec}$, followed by $60^{\circ} \mathrm{C}$ for $60 \mathrm{sec}$ and $95^{\circ} \mathrm{C}$ for $15 \mathrm{sec}$. Experiments were performed in duplicate and analyzed in triplicate; 16SrRNA was used as internal control. RT-qPCR data were analyzed using the relative quantitative $\left(2^{-\Delta \Delta \mathrm{Cq}}\right)$ method (26).

Statistical analysis. All data were analyzed using SPSS 19.0 (IBM Corp., Armonk, NY, USA) and are expressed as the mean \pm standard deviation. Statistical comparisons between groups were made using one-way analysis of variance followed by Fisher's LSD test. $\mathrm{P}<0.05$ was considered to indicate a statistically significant difference.

\section{Results}

Inhibition of $S$. aureus by BN. MICs of BN for C-4-4 and ATCC25923 strains were $32 \mu \mathrm{g} / \mathrm{ml}$. The MIC of VCM for the two strains was $0.5 \mu \mathrm{g} / \mathrm{ml}$. ATCC25923 was used as control strain; VCM as positive control.

Effects of BN on S. aureus biofilm formation in vitro. Biofilms were stained with crystal violet and FITC-ConA to study effects of BN on $S$. aureus biofilm formation. It was identified that compared with the control group, varying treatment times and concentrations of $\mathrm{BN}$ had an effect on biofilm formation, in addition to 6 and $12 \mathrm{~h} \mathrm{1/4} \mathrm{MIC} \mathrm{BN} \mathrm{(P<0.05;} \mathrm{Table} \mathrm{II).} \mathrm{With}$ an increase in $\mathrm{BN}$ concentration, the effect became more pronounced. The strongest decreases in OD measurements were observed with 1 MIC BN following 24 and $48 \mathrm{~h}$ (Table II).

FITC-ConA produces green fluorescence by combining with polysaccharides in the biofilm. The stronger the biofilm, 


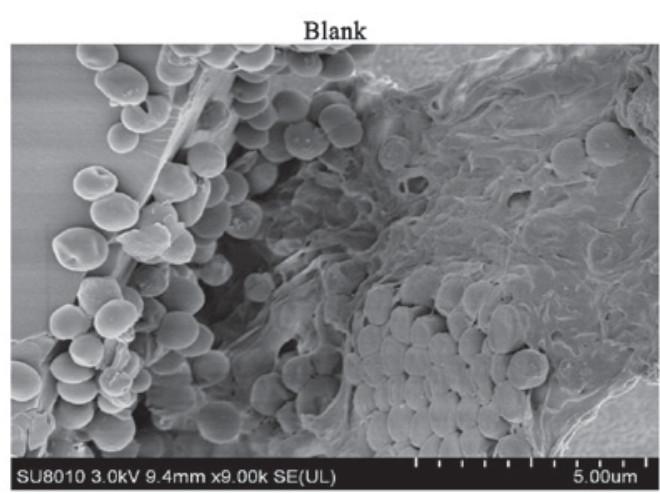

2 MIC BN

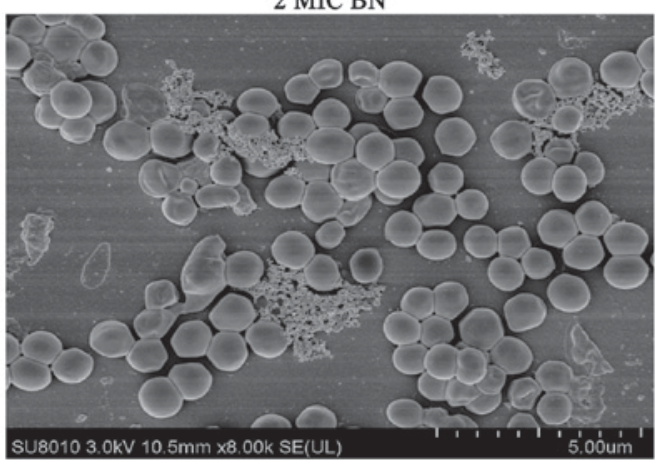

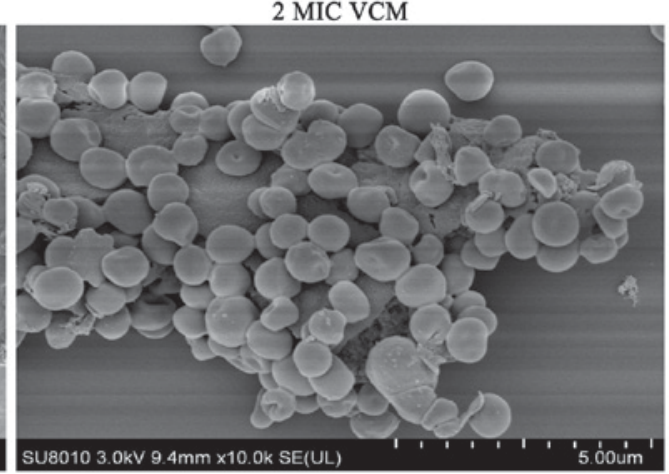

2 MIC VCM+2 MIC BN

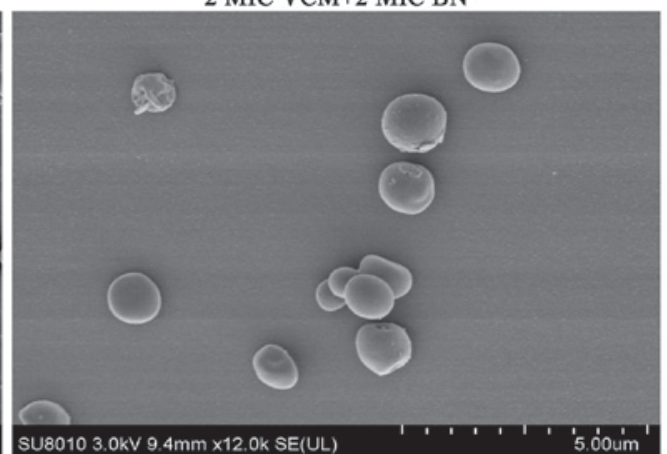

Figure 4. Biofilm topology by scanning electron microscopy (magnification, x8,000). Mature biofilms treated with BN (2 MIC) with or without VCM (2 MIC) and were cultivated at $37^{\circ} \mathrm{C}$ for $48 \mathrm{~h}$. Biofilms were gold coated prior to analysis. Scale bars, $5 \mu \mathrm{m}$. BN, brazilin; VCM, vancomycin; MIC, minimum inhibitory concentration.

the greater the green fluorescence (17). The current study used FITC-ConA-tagged fluorescence microscopy imaging to evaluate effects of $\mathrm{BN}$ on biofilm formation. The results revealed that $1 / 4,1 / 2$ and $1 \mathrm{MIC} B N$ for 12,24 and $48 \mathrm{~h}$ inhibited biofilm formation, whereas only minor effects were observed at $6 \mathrm{~h}$ (Fig. 1). At 48 h, the effect of $1 \mathrm{MIC}$ BN was most pronounced.

$B N$ combined with VCM damages the biofilm. The current study aimed to compare damaging effects of $\mathrm{BN}$ on $S$. aureus biofilms in the presence and absence of VCM, in order to assess synergistic effects. According to the crystal violet staining results, VCM alone only eradicated biofilms at 2 MIC following $48 \mathrm{~h}$. BN and $\mathrm{BN}+\mathrm{VCM}$ reduced biofilm formation in a concentration-dependent manner (Table III).

Fluorescence microscopy demonstrated that VCM had no marked effect on $S$. aureus biofilms compared with the control group, which exhibited large areas of green fluorescence. With an increase in $\mathrm{BN}$ concentration, mature biofilms were damaged; with increasing concentrations of $\mathrm{BN}+\mathrm{VCM}$, decreasing green fluorescence was observed (Fig. 2).

Biofilm bacterial CFU counts. VCM alone only reduced bacterial counts at $2 \mathrm{MIC}$ following $48 \mathrm{~h}$ treatment. $\mathrm{BN}$ at 1 or 2 MIC for 6 and $12 \mathrm{~h}$ and at 1/2, 1 or 2 MIC following $48 \mathrm{~h}$ was able to decrease bacterial counts. The most distinct effect was observed with $2 \mathrm{MIC}$ BN + 2MIC VCM at $48 \mathrm{~h}$ (Table IV).

Biofilm analysis using CLSM. SYTO9 stain labels live bacteria, whereas PI penetrates bacteria with damaged membranes. The results demonstrated that VCM was unable to damage the biofilm (Fig. 3). VCM groups exhibited mainly green fluorescence, representing live cells. Increasing concentrations of BN demonstrated an effect on the biofilm, exhibiting increasing red fluorescence, representing dead cells. BN + VCM groups exhibited increased levels of dead cells, represented by red fluorescence.

Biofilm damage assay by SEM. It was observed that biofilms treated with VCM exhibited large extracellular matrices and a large biofilm mass compared with the control group. BN was able to reduce the extracellular matrix and $\mathrm{BN}+\mathrm{VCM}$ groups exhibited increased effectiveness, illustrated by damaged biofilms and individual bacteria (Fig. 4). These findings indicate that $\mathrm{BN}$ may be able to penetrate biofilms.

Gene expression analysis. The current study aimed to measure mRNA levels in different samples using RT-qPCR, with $16 \mathrm{SrRNA}$ as internal control. Mature biofilms were treated with 1/2, 1 and 2 MIC BN for 6, 12, 24 and $48 \mathrm{~h}$. Treatment with 2 MIC BN for $12 \mathrm{~h}$, with 1 or $2 \mathrm{MIC}$ BN for $24 \mathrm{~h}$ or with $1 / 2,1$ or $2 \mathrm{MIC}$ BN for $48 \mathrm{~h}$ significantly lowered icaA expression levels compared with the untreated control $(\mathrm{P}<0.05$; Fig 5A). Transcription levels of icaR and agrA were significantly upregulated in $\mathrm{BN}$ treatment groups, compared with the control $(\mathrm{P}<0.05$; Fig. $5 \mathrm{~B}$ and $\mathrm{C})$. IcaR expression significantly increased compared with the control using $2 \mathrm{MIC}$ $\mathrm{BN}$ for $12 \mathrm{~h}, 1$ or $2 \mathrm{MIC} \mathrm{BN}$ for $24 \mathrm{~h}$ or $1 / 2,1$ or $2 \mathrm{MIC} \mathrm{BN}$ for $48 \mathrm{~h}$ ( $\mathrm{P}<0.05$; Fig. 5B). BN treatment significantly upregulated transcription levels of agrA following treatment times $\geq 12 \mathrm{~h}$ (Fig. 5C). No significant difference was observed following $\mathrm{BN}$ treatment for $6 \mathrm{~h}$ compared with the control. 

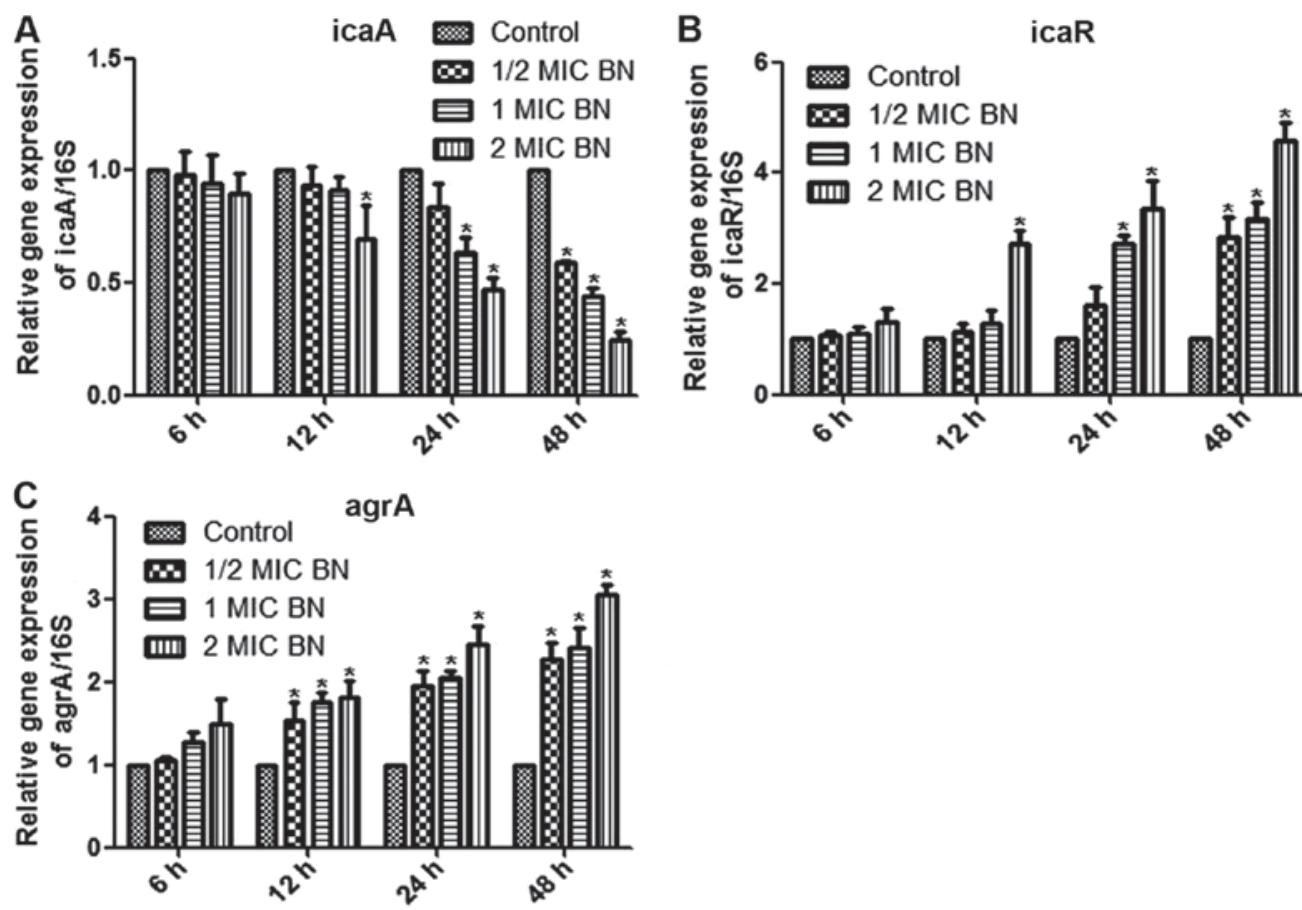

Figure 5. Gene expression using reverse transcription-quantitative polymerase chain reaction analysis. Relative gene expression of (A) icaA, (B) icaR and (C) $\arg \mathrm{A}$ in the presence of varying concentrations of $\mathrm{BN}(1 / 2,1$ and $2 \mathrm{MIC}) .16$ srRNA was used as internal control. "P<0.05 vs. control. Ica, intercellular adhesion; arg, accessory gene regulator; BN, brazilin; MIC, minimum inhibitory concentration.

\section{Discussion}

S. aureus biofilm development primarily includes four stages: Attachment, multiplication, maturation and dispersal (27). The principal mechanism of biofilm formation relies on secretion of extracellular polysaccharides (28). It was suggested that the matrix of biofilms may be responsible for increased resistance to antibiotics by acting as a diffusion barrier, causing chronic persistent infections that are difficult to cure $(29,30)$. At present, it is of great importance to identify methods for prevention and treatment of biofilm-associated infections. Studies have demonstrated that traditional herbal medicines were widely-sourced, had minimal side effects, strong pharmacological action and did not lead to bacterial drug resistance (15-17). The use of herbal medicines in the prevention and treatment of biofilm infections has a particular advantage. Traditional herbal medicine may not only inhibit the formation and destroy biofilms, but may enhance resistance to infections when combined with antibiotics (18).

The aim of the current study was to inhibit $S$. aureus biofilm formation, as assessed using crystal violet staining and FITC-ConA fluorescence microscopy. VCM was used as positive control. The results of the two experiments were similar: $\mathrm{BN}$ inhibited biofilm formation in vitro in a dose-dependent manner and the greatest effect was obtained with 2 MIC BN following 24 and $48 \mathrm{~h}$. The results were similar to those reported by Lázaro-Díez et al (6).

At present, VCM is the most commonly administered drug for S. aureus biofilm-associated infections (31). However, there is cause for concern due to recent developments of VCM-intermediate $S$. aureus and VRSA strains (10). The question of how to reduce the generation of drug-resistant strains and improve the effectiveness of VCM in biofilm infections requires further research. Combining Chinese herbal medicines with VCM is a possible novel approach to treating biofilm-associated infections.

In the current study, to identify synergistic effects of BN combined with VCM on $S$. aureus biofilms, crystal violet staining, fluorescence microscopy, CFU counting, CLSM and SEM were used. This array of methods demonstrated that BN and BN + VCM generally enhanced the destruction of biofilms in a concentration-dependent manner; 2 MIC BN + 2 MIC VCM exhibited the most notable effects, whereas VCM alone was ineffective. Chen et al (32) studied inhibitory effects of baicalein on $S$. aureus biofilm formation using these methods, achieving outcomes similar to experimental results presented here.

In summary, VCM may not disrupt a biofilm when used alone, whereas $\mathrm{BN}$ may be able to do so. $\mathrm{BN}$ may reduce the production of the extracellular polymeric matrix or it may penetrate the biofilm to increase its permeability to VCM. In the current study, BN and VCM exhibited a synergy in eradicating the biofilm.

S. aureus biofilm formation is a complex dynamic process, involving numerous regulatory mechanisms, including polysaccharide intercellular adhesion (PIA), extracellular DNA, global regulatory factors and the QS system (28). PIA is synthesized by the ica locus and was first identified in the epidermis $S$. aureus biofilm (33). The ica locus includes icaR (regulatory) and icaADBC (biosynthetic), which affect biofilm formation (34). PIA is the primary component of the Gram-positive bacterial biofilm matrix, has the largest influence on biofilm formation and is a well-studied component (35). Studies have demonstrated that icaR acts as a repressor gene and PIA generation increases following knockout of icaR $(34,36)$. In addition, PIA expression is repressed by tcaR, a transcriptional regulator of the teicoplanin-associated locus. The protein regulator of biofilm 
formation augments ica gene expression, PIA production and biofilm formation (37-39). In addition, Pamp et al (40) demonstrated that Spx, a global regulator of stress response genes, inhibits biofilm formation, potentially by modulating icaR. When strains were cultured in anaerobic environments, the $S$. aureus respiratory response regulator $\mathrm{SrrAB}$ was responsible for PIA, promoting biofilm formation (41). Regulation of the ica locus influences production of PIA and formation of biofilms, making it a novel potential therapeutic target.

In the current study, mature biofilms were treated with $\mathrm{BN}$ for $6,12,24$ and $48 \mathrm{~h}$. It was observed that $\mathrm{BN}$ was able to downregulate icaA expression. Transcription levels of icaR were significantly upregulated. BN may regulate icaA and icaR expression in order to inhibit the synthesis of PIA and to reduce the secretion of extracellular polysaccharides.

The $S$. aureus biofilm QS system includes the agr system and luxS/AI-2 (42). The agr QS system has been well-researched and includes agrBDCA and RNAIII (43). The autoinducer aryl hydrocarbon receptor-interacting protein (AIP) is generated from ArgD precursors and is secreted via the AgrB membrane protein (43). When the extracellular AIP concentration reaches a threshold, agrC is phosphorylated. AgrC phosphorylation activates molecular agrA, which in turn activates RNAIII (44). A previous study demonstrated that when the agr system is mutated, biofilm formation is enhanced (45). The agr system promotes biofilm maturation. The addition of AIP to the mature biofilm may additionally promote biofilm dispersal (45).

The current study demonstrated that $\mathrm{BN}$ was able to upregulate transcription levels of agrA. BN may inhibit gene expression of the QS signaling molecule agrA and block RNAIII to prevent biofilm formation. However, the question of whether BN inhibits the QS system requires further analysis.

In conclusion, $\mathrm{BN}$ was able to damage mature biofilms potentially by enhancing VCM permeability and through potential inhibition of the secretion of extracellular polysaccharides and the QS system. BN is a potential novel treatment against $S$. aureus biofilm-associated infections. The mechanisms involved require further analysis and experimentation in vivo.

\section{Acknowledgements}

The authors would like to thank Dr Xun Min, Dr Tao Zhang and Dr Yingbiao Tian from the Zunyi Medical University (Zunyi, China) for their expert advice and technical assistance throughout the study.

\section{Funding}

No funding was received.

\section{Availability of data and materials}

The datasets used and/or analyzed during the current study are available from the corresponding author on reasonable request.

\section{Authors' contributions}

ZHC, XM, YBT and TZ conceived and designed the study. DP, ALC, XLC and BS performed the experiments. DP, ZLD,
ALC, XLC and HY analyzed the data. DP, ZLD, XLC and YBT contributed reagents, materials and analysis tools. DP and $\mathrm{ZHC}$ wrote the paper. $\mathrm{TZ}$ revised the manuscript.

\section{Ethics approval and consent to participate}

The present study was approved by the Ethics Committee of the Affiliated Hospital of Zunyi Medical University and written informed consent was obtained prior to the collection of all samples.

\section{Patient consent for publication}

Not applicable.

\section{Competing interests}

The authors declare that they have no competing interests.

\section{References}

1. Yu L, Hisatsune J, Hayashi I, Tatsukawa N, Sato'o Y, Mizumachi E, Kato F, Hirakawa H, Pier GB and Sugaia M: A Novel Repressor of the ica locus discovered in clinically isolated super-biofilm-elaborating Staphylococcus aureus. MBio 8: pii:e02282-16, 2017.

2. Yoshii Y, Okuda KI, Yamada S, Nagakura M, Sugimoto S, Nagano T, Okabe T, Kojima H, Iwamoto T, Kuwano K and Mizunoe Y: Norgestimate inhibits staphylococcal biofilm formation and resensitizes methicillin-resistant Staphylococcus aureus to $\beta$-lactam antibiotics. NPJ Biofilms Microbiomes 3: 18 , 2017.

3. Sollid JU, Furberg AS, Hanssen AM and Johannessen M: Staphylococcus aureus: Determinants of human carriage. Infect Genet Evol 21: 531-541, 2014.

4. Kong C, Chee CF, Richter K, Thomas N, Abd Rahman N and Nathan S: Suppression of Staphylococcus aureus biofilm formation and virulence by a benzimidazole derivative, UM-C162. Sci Rep 8: 2758, 2018.

5. Waryah CB, Wells K, Ulluwishewa D, Chen-Tan N, Gogoi-Tiwari J, Ravensdale J, Costantino P, Gökçen A, Vilcinskas A, Wiesner J and Mukkur T: In vitro antimicrobial efficacy of tobramycin against Staphylococcus aureus biofilms in combination with or without DNase I and/or Dispersin B: A preliminary investigation. Microb Drug Resist 23: 384-390, 2017.

6. Lázaro-Díez M, Remuzgo-Martínez S, Rodríguez-Mirones C, Acosta F, Icardo JM, Martínez-Martínez L and Ramos-Vivas J: Effects of subinhibitory concentrations of ceftaroline on methicillin-resistant Staphylococcus aureus (MRSA) biofilms. PLoS One 11: e0147569, 2016

7. Feuillie C, Formosa-Dague C, Hays LM, Vervaecka O, Derclayea S, Brennanc MP, Fosterb TJ, Geogheganb JA and Dufrêne YF: Molecular interactions and inhibition of the staphylococcal biofilm-forming protein SdrC. Proc Natl Acad Sci USA 114: 3738-3743, 2017.

8. Howlin RP, Brayford MJ, Webb JS, Cooper JJ, Aiken SS and Stoodley P: Antibiotic-loaded synthetic Calcium sulfate beads for prevention of bacterial colonization and biofilm formation in periprosthetic infections. Antimicrob Agents Chemother 59: 111-120, 2015.

9. Koch G, Yepes A, Förstner KU, Wermser C, Stengel ST, Modamio J, Ohlsen K, Foster KR and Lopez D: Evolution of resistance to a last-resort antibiotic in Staphylococcus aureus via bacterial competition. Cell 158: 1060-1071, 2014.

10. Howden BP, Davies JK, Johnson PD, Stinear TP and Grayson ML: Reduced vancomycin susceptibility in Staphylococcus aureus, including vancomycin-intermediate and heterogeneous vancomycin-intermediate strains: Resistance mechanisms, laboratory detection, and clinical implications. Clin Microbiol Rev 23: 99-139, 2016.

11. Kiedrowski MR and Horswill AR: New approaches for treating staphylococcal biofilm infections. Ann N Y Acad Sci 1241: 104-121, 2011. 
12. Bhattacharya M, Wozniak DJ, Stoodley P and Hall-Stoodley L: Prevention and treatment of Staphylococcus aureus biofilms. Expert Rev Anti Infect Ther 13: 1499-1516, 2015.

13. Salem AH, Elkhatib WF and Noreddin AM: Pharmacodynamic assessment of vancomycin-rifampicin combination against methicillin resistant Staphylococcus aureus biofilm: A parametric response surface analysis. J Pharma Pharmacol 63: 73-79, 2011.

14. Olson ME, Slater SR, Rupp ME and Fey PD: Rifampicin enhances activity of daptomycin and vancomycin against both a polysaccharide intercellular adhesin (PIA)-dependent and -independent Staphylococcus epidermidis biofilm. J Antimicrob Chemother 65: 2164-2171, 2010.

15. Fontaine BM, Nelson K, Lyles JT, Jariwala PB, García-Rodriguez JM, Quave CL and Weinert EE: Identification of ellagic acid rhamnoside as a bioactive component of a complex botanical extract with anti-biofilm activity. Front Microbiol 8: 496, 2017.

16. Fu B, Wu Q, Dang M, Bai D, Guo Q, Shen L and Duan K: Inhibition of pseudomonas aeruginosa biofilm formation by traditional chinese medicinal herb herba patriniae. Biomed Res Int 2017: 9584703, 2017

17. Luo J, Dong B, Wang K, Cai S, Liu T, Cheng X, Lei D, Chen Y, Li Y, Kong J and Chen Y: Baicalin inhibits biofilm formation, attenuates the quorum sensing-controlled virulence and enhances Pseudomonas aeruginosa clearance in a mouse peritoneal implant infection model. PLoS One 12: e0176883, 2017.

18. García-Heredia A, García S, Merino-Mascorro JÁ, Feng P and Heredia N: Natural plant products inhibits growth and alters the swarming motility, biofilm formation, and expression of virulence genes in enteroaggregative and enterohemorrhagic Escherichia coli. Food Microbiol 59: 124-132, 2016

19. Kim J, Lee HK, Chang TS, Kang KS and Hwang GS: Inhibitory effect of brazilin on osteoclast differentiation and its mechanism of action. Int Immunopharmacol 29: 628-634, 2015.

20. Gao XJ, Wang TC, Zhang ZC, Cao YG, Zhang NS and Guo MY: Brazilin plays an anti-inflammatory role with regulating Toll-like receptor 2 and TLR 2 downstream pathways in Staphylococcus aureus-induced mastitis in mice. Int Immunopharmacol 27: $130-137,2015$

21. Dan P, Zhou X and Chen Z: Research progress on mechanism and treatment of Staphylococcus aureus biofilms. Molecular Biomedicine 23: 3745-3749, 2017.

22. Nirmal NP and Panichayupakaranant P: Antioxidant, antibacterial, and anti-inflammatory activities of standardized brazilin-rich Caesalpinia sappan extract. Pharm Biol 53: 1339-1343, 2015.

23. Yan Y, Chen YC, Lin YH, Guo J, Niu ZR, Li L, Wang SB Fang LH and Du GH: Brazilin isolated from the heartwood of Caesalpinia sappan L induces endothelium-dependent and-independent relaxation of rat aortic rings. Acta Pharmacol Sin 36: 1318-1326, 2015

24. Clinical and Laboratory Standards Institute: Methods for dilution antimicrobial susceptibility tests for bacteria that grow aerobically; approved standard, 9th ed CLSI document M7-A9. Clinical and Laboratory Standards Institute, Wayne, PA, 2012.

25. Shi SF, Jia JF, Guo XK, Zhao YP, Chen DS, Guo YY and Zhang XL: Reduced Staphylococcus aureus biofilm formation in the presence of chitosan-coated iron oxide nanoparticles. Int J Nanomedicine 11: 6499-6506, 2016.

26. Livak KJ and Schmittgen TD: Analysis of relative gene expression data using real-time quantitative PCR and the 2(-Delta Delta C(T)) method. Methods 25: 402-408, 2001.

27. Balasubramanian S, Othman EM, Kampik D, Stopper H, Hentschel U, Ziebuhr1 W, Oelschlaeger1 TA and Abdelmohsen UR: Marine sponge-derived Streptomyces sp. SBT343 extract inhibits Staphylococcal biofilm formation. Front Microbiol 8: 236, 2017.

28. Sugimoto S, Sato F, Miyakawa R, Chiba A, Onodera S, Hori S and Mizunoe Y: Broad impact of extracellular DNA on biofilm formation by clinically isolated Methicillin-resistant and-sensitive strains of Staphylococcus aureus. Sci Rep 8: 2254, 2018
29. Singh AK, Prakash P, Achra A, Singh GP, Das A and Singh RK: Standardization and classification of In vitro biofilm formation by clinical isolates of Staphylococcus aureus. J Glob Infect Dis 9: 93-101, 2017.

30. Brooks JL and Jefferson KK: Staphylococcal biofilms: Quest for the magic bullet. Adv Appl Microbiol 81: 63-87, 2012.

31. van Hal SJ, Lodise TP and Paterson DL: The clinical significance of vancomycin minimum inhibitory concentration in Staphylococcus aureus infections: A systematic review and meta-analysis. Clin Infect Dis 54: 755-771, 2012.

32. Chen Y, Liu T, Wang K, Hou C, Cai S, Huang Y, Du Z, Huang H, Kong J and Chen Y: Baicalein inhibits Staphylococcus aureus biofilm formation and the quorum sensing system in vitro. PLoS One 11: e0153468, 2016

33. O'gara JP: Ica and beyond: Biofilm mechanisms and regulation in Staphylococcus epidermidis and Staphylococcus aureus. FEMS Microbiol Lett 270: 179-188, 2007.

34. Figueiredo AMS, Ferreira FA, Beltrame CO and Côrtes MF The role of biofilms in persistent infections and factors involved in ica-independent biofilm development and gene regulation in Staphylococcus aureus. Crit Rev Microbiol 43: 602-620, 2017.

35. Ma R, Qiu S, Jiang Q, Sun H, Xue T, Cai G and Sun B: AI-2 quorum sensing negatively regulates rbf expression and biofilm formation in Staphylococcus aureus. Int J Med Microbiol 307: 257-267, 2017.

36. Gupta A, Mishra S, Singh S and Mishra S: Prevention of IcaA regulated poly $\mathrm{N}$-acetyl glucosamine formation in Staphylococcus aureus biofilm through new-drug like inhibitors: In silico approach and MD simulation study. Microb Pathog 110: 659-669, 2017.

37. Skurnik D, Cywes-Bentley C and Pier GB: The exceptionally broad-based potential of active and passive vaccination targeting the conserved microbial surface polysaccharide PNAG. Expert Rev Vaccines 15: 1041-1053, 2016.

38. Formosa-Dague C, Feuillie C, Beaussart A, Derclaye S, Kucharíková S, Lasa I, Van Dijck P and Dufrêne YF: Sticky matrix: Adhesion mechanism of the staphylococcal polysaccharide intercellular adhesin. ACS Nano 10: 3443-3452, 2016.

39. Doulgeraki AI, Di Ciccio P, Ianieri A and Nychas GE: Methicillin-resistant food-related Staphylococcus aureus: A review of current knowledge and biofilm formation for future studies and applications. Res Microbiol 168: 1-15, 2017.

40. Pamp SJ, Frees D, Engelmann S, Hecker M and Ingmer H: Spx is a global effector impacting stress tolerance and biofilm formation in Staphylococcus aureus. J Bacteriol 188: 4861-4870, 2006.

41. Kinkel TL, Roux CM, Dunman PM and Fang FC: The Staphylococcus aureus SrrAB two-component system promotes resistance to nitrosative stress and hypoxia. MBio 4: e00696-e13, 2013.

42. Coelho LR, Souza RR, Ferreira FA, Guimarães MA, Ferreira-Carvalho BT and Figueiredo AM: Agr RNAIII divergently regulates glucose-induced biofilm formation in clinical isolates of Staphylococcus aureus. Microbiology 154: 3480-3490, 2008.

43. Atwood DN, Beenken KE, Loughran AJ, Meeker DG, Lantz TL, Graham JW, Spencer HJ and Smeltzer MS: XerC contributes to diverse forms of Staphylococcus aureus infection via agr-dependent and agr-independent pathway. Infect Immun 84: 1214-1225, 2016.

44. Le KY and Otto M: Quorum-sensing regulation in staphylococci-an overview. Front Microbiol 6: 1174, 2015.

45. Boles BR and Horswill AR: Agr-mediated dispersal of Staphylococcus aureus biofilms. PLoS Pathog 4: e1000052, 2008.

This work is licensed under a Creative Commons Attribution-NonCommercial-NoDerivatives 4.0 International (CC BY-NC-ND 4.0) License. 